\title{
Percutaneous local ablation of unifocal subclinical breast cancer: clinical experience and preliminary results of cryotherapy
}

\author{
Guglielmo Manenti • Tommaso Perretta • Eleonora Gaspari • Chiara A. Pistolese • \\ Lia Scarano - Elsa Cossu • Elena Bonanno - Oreste C. Buonomo - Giuseppe Petrella • \\ Giovanni Simonetti $\cdot$ Salvatore Masala
}

Received: 14 February 2011 / Accepted: 2 June 2011 / Published online: 17 June 2011

(C) European Society of Radiology 2011

\begin{abstract}
Objectives To assess the ablative effectiveness, the oncological and cosmetic efficacy of image-guided percutaneous cryoablation in the treatment of single breast nodules with subclinical dimensions after identification with ultrasonography (US), mammography, magnetic resonance (MRI) and characterization by vacuum assisted biopsy.

Materials Fifteen women with a mean age of $73 \pm 5$ years (range 64-82 years) and lesion diameter of $8 \pm 4 \mathrm{~mm}$ were undergoing cryotherapy technology with a single probe under US-guidance associated with intra-procedural lymphnode mapping and excision of the sentinel node. All the patients underwent surgical resection (lumpectomy) from 30 to 45 days after the percutaneous ablation.

Results The iceball size generated by the cryoprobe during the procedure at minus $40^{\circ} \mathrm{C}$ was $16 \times 41 \mathrm{~mm}$. In 14 of the 15 patients was observed a complete necrosis of the cryoablated lesion both in post-procedural MRI follow-up and
\end{abstract}

G. Manenti $(\bowtie) \cdot$ T. Perretta $\cdot$ E. Gaspari $\cdot$ C. A. Pistolese $\cdot$

L. Scarano $\cdot$ E. Cossu $\cdot$ G. Simonetti $\cdot$ S. Masala

Department of Diagnostic Imaging and Interventional Radiology,

Molecular Imaging and Radiotherapy,

University Hospital "Tor Vergata",

viale Oxford 81 ,

00133 Rome, Italy

e-mail: guggi@tiscali.it

O. C. Buonomo • G. Petrella

Department of General Surgery Division,

University Hospital "Tor Vergata",

viale Oxford 81,

00133 Rome, Italy

\section{E. Bonanno}

Department of Biopathology, University Hospital "Tor Vergata", viale Oxford 81 ,

00133 Rome, Italy anatomo-pathological evaluation after surgical resection. In one case there was a residual disease in post-procedural MRI and postoperative histological examination, probably justified by an incorrect positioning of the probe.

Conclusion The percutaneous cryoablation as a "minimally invasive" technique can provide excellent oncological and cosmetic results on selected cases handled by experienced operators by using the tested devices.

Keywords Cryotherapy $\cdot$ Percutaneous ablation $\cdot$ Magnetic resonance imaging $\cdot$ Breast cancer $\cdot$ Minimally invasive

\section{Introduction}

The reduction in breast cancer mortality is attributable to the combined effect of an early diagnosis and a more effective treatment. Breast-conserving surgery has been shown to provide good functional and cosmetic results without altering the survival rate of patients $[1,2]$. Next step in cancer research is projected to the identification of instrumental approaches as effective as surgery, but minimally invasive, such as to be cosmetically more acceptable to patients. The breast is a suitable organ for percutaneous treatment, being superficially localized in the chest and covered only by skin, in the absence of visceral organs between them. Breast cancer is also a major cause of morbidity and mortality in older women, affecting up to $30 \%$ of the over-seventy years old patients, that with the increase in life expectancy, is likely to exceed $30 \%$ in the next decade [3, 4]. Patients "over 70" are subject to less aggressive treatments for the common presence of comorbidity. The aim of this study was to evaluate the functional outcome (quality of life - therapy satisfaction), 
the cosmetic results and the ablative effectiveness of cryolumpectomy in the treatment of single malign breast nodules with clinical dimensions close to $1 \mathrm{~cm}$, previously identified by ecotomography-mammography and MRI and characterized by vacuum assisted biopsy (VAB). The feasibility study is supported by the validation tests with high field (3T) MRI study aimed to determine the role of this method in quantifying the results.

\section{Materials and methods}

Patient characteristics and enrollment

The study was approved by our institutional review board. All patients gave written informed consent. Fifteen postmenopausal women (mean age, $73 \pm 5$ years [SD]; range 6482 years) with breast cancer were included in a study period between February and July 2010. The inclusion criteria were as follows: biopsy-proved ductal invasive unifocal breast cancer $1 \mathrm{~cm}$ or smaller, visible at both ultrasound (US) and magnetic resonance (MR) imaging, located at least $1 \mathrm{~cm}$ from the skin and $1 \mathrm{~cm}$ from the chest wall at US examination. The maximum diameter of each lesion was $8 \pm 4 \mathrm{~mm}$ (range 4$12 \mathrm{~mm}$ ). The distance of the lesions from the skin plane varied from 1 to $3 \mathrm{~cm}$. Patient exclusion criteria were: multifocal or multicentric neoplasia, lobular neoplasia, intraductal carcinoma, retro-areolar tumours and other forms of infiltrating cancer excluding ductal cancer. Patients with medical contraindications to MR imaging and those with anticoagulant drugs treatment were not considered eligible.

\section{Breast US}

The affected breast was imaged to include the known tumour and the surrounding breast tissue (in the same quadrant) by using a 5-12 MHz transducer and a US unit (ATL HDI 5000; Philips Medical Systems, Best, the Netherlands).

\section{Breast MR imaging}

Breast MR imaging examinations were performed with the patient prone in a 3.0-T system (Achieva; Philips Healthcare, Best, the Netherlands). A dedicated sensitivity-encoding breast coil was used for RF signal reception. Axial T2-weighted and T1-weighted images were obtained. The dynamic series consisted of a T1-weighted three-dimensional fast-field-echo sequence (repetition time msec/echo time msec, 5.1/2.4; flip angle, $20^{\circ}$ ). A total of eight dynamic acquisitions, with a temporal resolution of $70 \mathrm{~s}$ for a single dynamic acquisition, were performed. Each dynamic acquisition consisted of 60 $2.5 \mathrm{~mm}$-thick sections (gap, 0 ) with a $256 \times 512$ matrix and a field of view of $330 \mathrm{~mm}$ (adjusted to the size of the breast).
Contrast agent bolus injection consisted of $0.2 \mathrm{mmol}$ gadopentetate dimeglumine (Magnevist; Schering, Berlin, Germany) per kilogram of body weight administered at an injection rate of $2.5 \mathrm{~mL} / \mathrm{sec}$. Patients underwent three MR imaging examinations: 1 week before the procedure, 1 week after the procedure, and 4 weeks after the procedure. .

\section{Vacuum assisted biopsy: VAB}

Lesions were characterized with vacuum biopsy systems Vacora-Bard (Bard Biopsy Systems, Tempe, AZ, USA) or Mammotome Breast Biopsy system (Ethicon Endo-Surgery, Cincinnati, $\mathrm{OH}, \mathrm{USA}$ ) under ultrasound guidance for standard histological and immunohistochemical parameters (receptors for estrogen, progesterone and HER-2/neu). This procedure involves the use of ultrasound guidance; needles of different caliber are used with a coaxial system of collection/aspiration that allows multiple samples through a single percutaneous insertion.

\section{Cryoablation procedure}

The procedure was performed in the "convertible" angiography room. The devices were placed percutaneously by "real time" ultrasound guidance using an ultrasound probe at 5-12 MHz (ATL 5000, Philips, Best, The Netherlands). After disinfecting the skin of the breast, $10 \mathrm{ml}$ of xilocaine $2 \%$ were administered subcutaneously, along the path of the probe and in the target area. After skin incision with a scalpel ( $3 \mathrm{~mm})$, one cryo-probe (1.47 mm diameter 17 Gauge Isotherms; IceRod model - Galil Medical, Yokneam, Israel) and two thermocouples for temperature monitoring were positioned (Fig. 1). The device is set with the tip of the exposed needle at $2-5 \mathrm{~mm}$ from the distal margin of the lesion. A bland intravenous sedation was always effected with awake and collaborating patient during the procedure. If the distance between the lesion and the skin profile and/or muscle is equal to $1 \mathrm{~cm}$, sterile saline solution heated at $40^{\circ} \mathrm{C}$ was injected to avoid surrounding skin and chest wall damage. Two cycles, each one with two cooling phases alternated by an active heating phase as long as $5 \mathrm{~min}$, were completed with the aim to produce a suitable volume of necrosis. Further heating was than necessary to dissolve the iceball (Fig. 2). At the end of the procedure probe and thermocouples were removed without the need for stitches, and a sterile patch with compressive bandage was packed. In the same session surgical excision of the sentinel lymph node for subsequent histological evaluation was performed. Patients were educated on the necessity to report post-procedural symptoms at following weekly clinical follow-up. The appearance of the skin, the size of the cryo-lesion and the procedural comfort of the patient were carefully recorded at the procedure end. An oral wide-spectrum antibiotic therapy was prescribed for the 5 days following the procedure. 

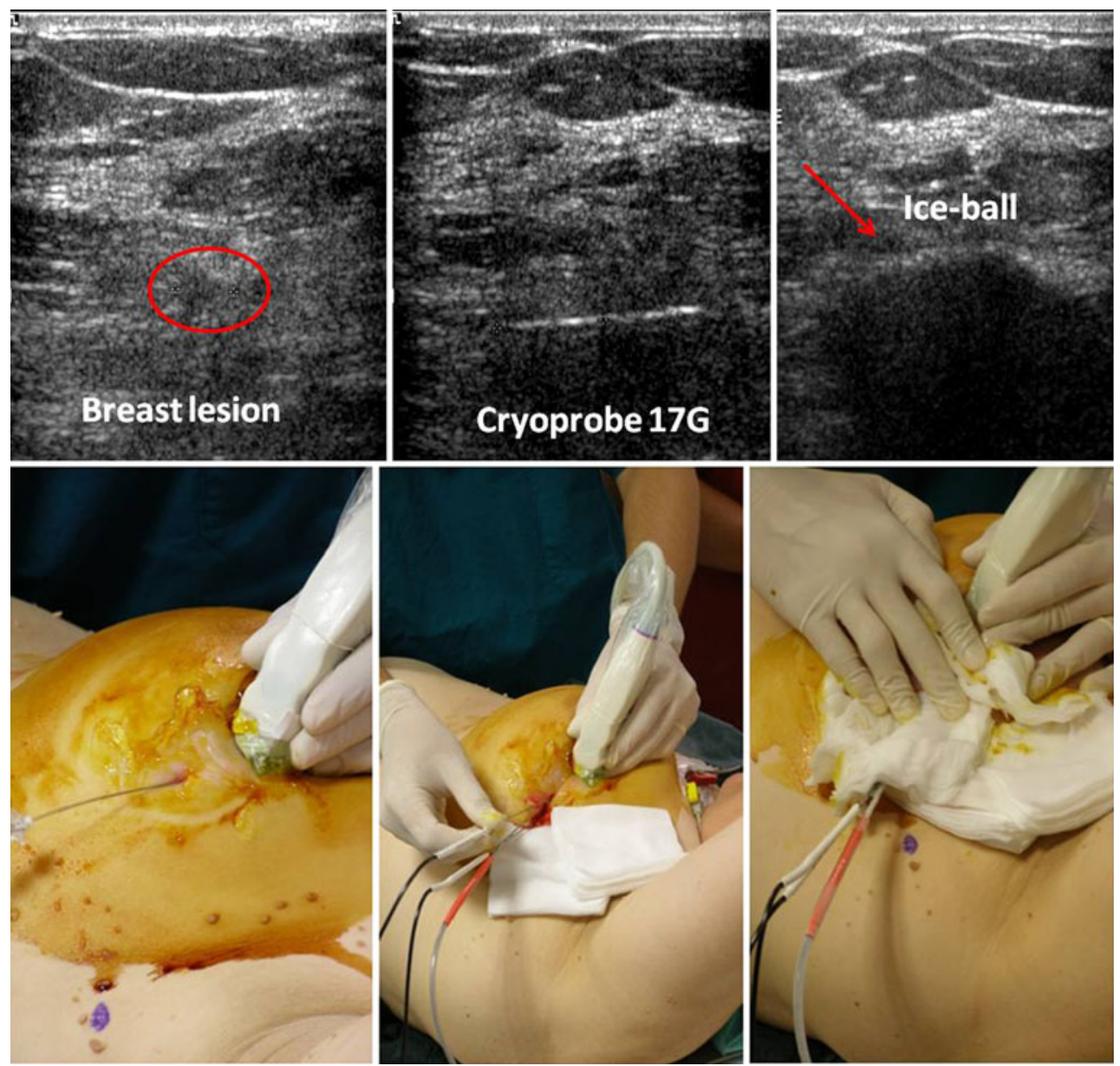

Fig. 1 Cryoablation procedure: this type of equipment is based on the thermodynamic properties of some gases (argon and helium), which on argon expansion (change from a smaller volume to a larger volume) from an high pressure (3000 psi) to low environment pressure remove heat from the external environment or, inversely, release heat (helium) according to the Joule-Thompson effect. The devices were placed percutaneously by "real time" ultrasound guidance using an ultrasound probe at 5-12 MHz. After skin incision with a scalpel (3 $\mathrm{mm})$, the cryoprobe (17 G Isotherms) and two thermocouples for temperature monitoring were positioned. The compressed argon gas passes through the needle during cryoablation, cooling the tip and generating the iceball in the adjacent tissue because of adiabatic effect

\section{Surgery}

All the patients underwent lumpectomy about 34 days after the percutaneous ablation procedure (range, 30-45 days).

\section{Histopathologic analysis}

Breast biopsy was performed to characterize the neoplasia before treatment (Fig. 3). Samples were formalin fixed and paraffin embedded. Histological sections were stained with haematoxylin eosin for tumour staging. Predictive and prognostic markers were studied by immunohistochemistry testing ER (Ventana ${ }^{1}$ CONFIRM anti-Estrogen Receptor
(Joule-Thompson phenomenon). The compressed helium gas expanding in the coaxial needle heats the exposed tip of the probe arresting the progression of the procedure and/or alternatively dissolving the iceball at the end of the second cooling cycle by restoring the electrode-tissue interface. The gas flow is finely regulated by a computerized system, so that the temperatures of cryoprobes are optimized within a few seconds. The expanded gas is recirculated through the cryogenic system to the outer lumen of the probe and therefore, released from a reservoir directly into the environment. The application of hot patches on the skin adjacent to the probe has also significantly reduced the after treatment skin rashes incidence by achieving a good definitive cosmetics

ER SP1), PR (Ventana CONFIRM anti-PR 1E2), c-erb-B2 (PATHWAY HER2- Ventana), the proliferative activity of tumour cells was evaluated by Ki-67 index (Ventana CONFIRM Ki-67).

\section{Cosmetic assessment}

The post-ablation cosmetics was assessed respectively at the end of the ablation (Time 0 ) and at 1 month from the procedure, prior to the surgical resection of the tumour (Time I). The cosmetic assessment was expressed according

\footnotetext{
${ }^{1}$ Ventana Medical Systems, Inc Tucson Arizona
} 


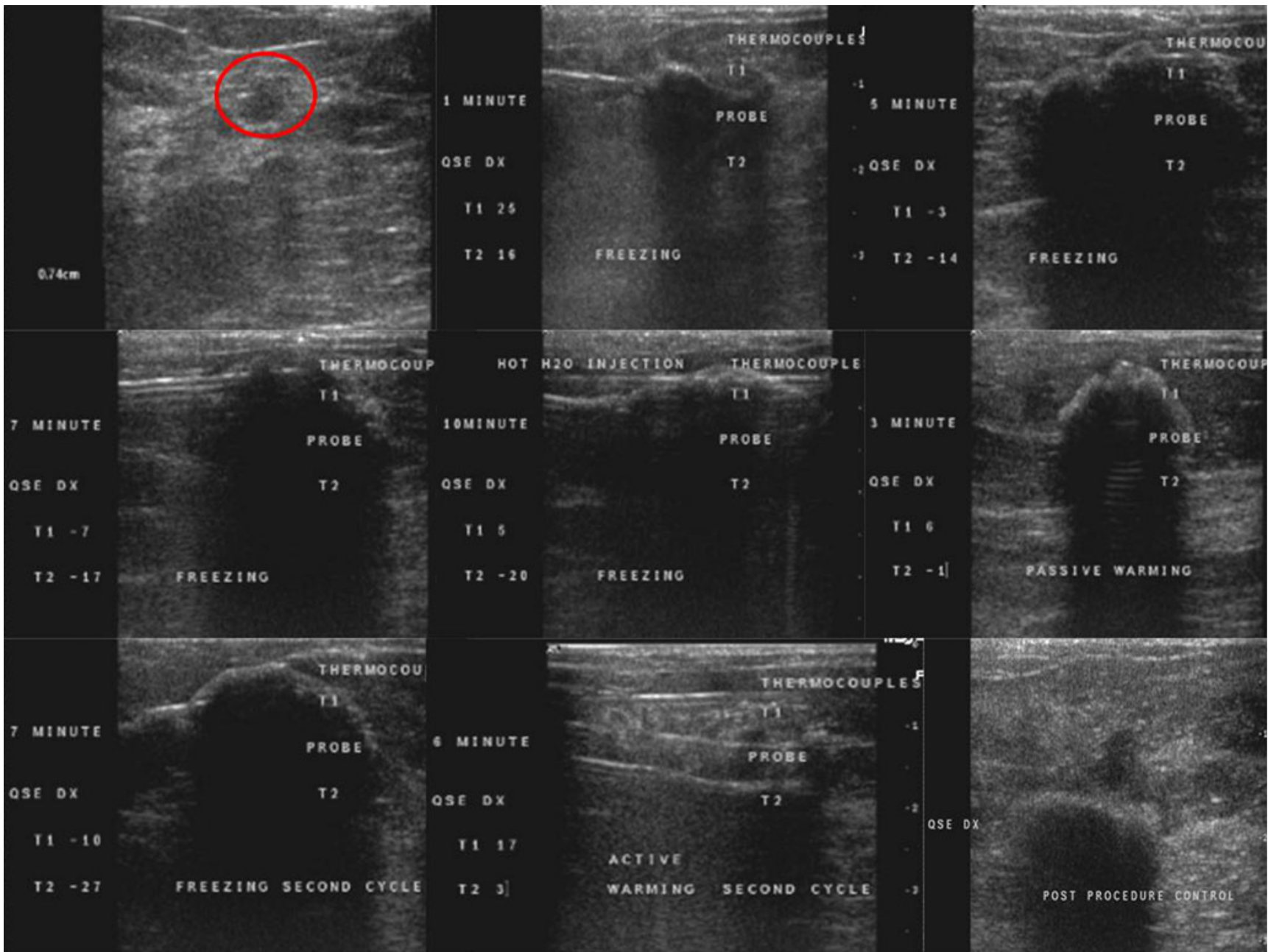

Fig. 2 Percutaneous cryoablation procedure ultrasound-guidance: two cycles, each one with two cooling phases alternated by an active heating phase, were completed with the aim to produce a suitable volume of necrosis. Ultrasound identifies the cryolesions in terms of characteristically hyperechoic iceball with anechoic treatment area for all the procedure duration or with a "comet" pattern, requiring as a condition, the distal centering of peripheral edge of the lesion. The treated tumor becomes fully visible at the end of treatment, following the iceball thaw out. Starting the procedure, the gas begins to flow

to the presence or absence of 4 parameters: appreciable nodular thickening on palpation, skin rash, skin bruising, skin hyperpigmentation. The absence of such signs is interpreted as an optimal cosmetic result; the presence of 1 or 2 marks as a good cosmetic result.

\section{Follow up}

The lesions treated with ultrasound guided percutaneous ablation were evaluated weekly by serial ultrasound examinations up to four weeks since the procedure. MRI was performed respectively one and four weeks after the cryoablation procedure by means of dynamic post-contrast sequences (Figs. 4, 5, 6). All the patients were followed at least with inside the needle tip, the tissue surrounding it goes on transition phase by creating a forehead freezing. The freezing interface extends progressively into the tissue volume at a rate that varies according to the composition and vascularity of the tissue. The forehead freezing stops when the argon flow inside the needle is blocked or if blood flow in the periphery is likely to carry on the same quantity of heat absorbed by the needle itself (thermodynamic equilibrium). Further heating was than necessary to dissolve the iceball

one breast US, mammography and MRI at 6 months after radiotherapy, performed in the period within 3 months after surgery.

\section{Statistical analysis}

All data analysis was performed using the Statistical Package for the Social Sciences Windows, version 15.0 (SPSS, Chicago, Illinois, USA). Descriptive statistics consisted in the mean \pm SD for variables with Gaussian distributions (after confirmation with histograms and the Kolgomorov-Smirnov test) or median (min-max) for categorical parametric and non-parametric data. Comparison among for data paired were carried out with $t$-test or 
Fig. 3 Histological examination of formalin fixed lumpectomy specimens was conducted according to the following protocol: a Orientation of the lumpectomy macroscopic sample according to MRI images; b Section of the entire sample for the gross specimen assessment; c-d Identification and collection of hemorrhagic/ necrotic macroscopically evident areas and all the relevant pathologic areas at contrastenhanced MRI; Collection and inclusion of all the disease areas identified; e-f-g-h) Histological examination with hematoxylineosin and immunohistochemistry to assess the presence of epithelial cells (Ventana Confirm anti-cytokeratin). Blind assessment carried out by two pathologists for histological preparation
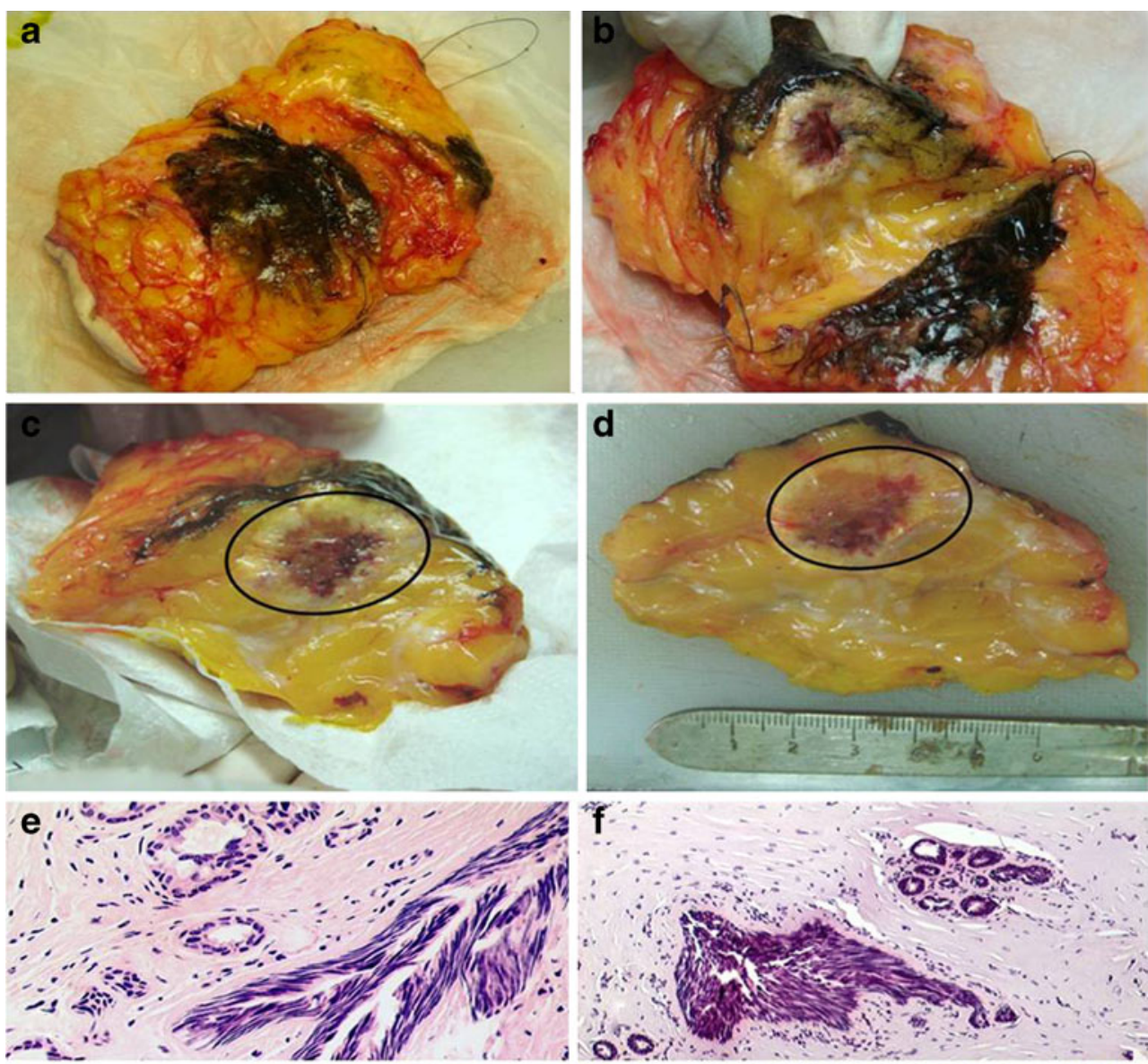

g

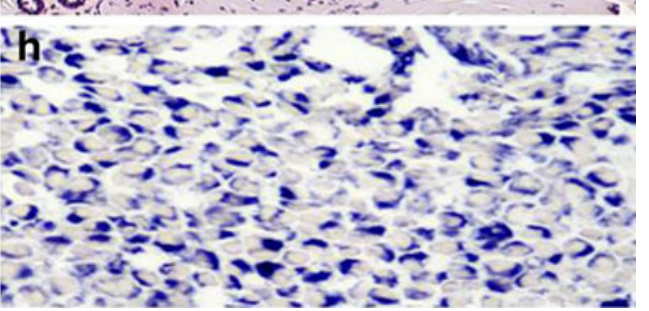

Fig. 4 a-a') Axial T2 MRI image: pre-cryoablation image with focal lesion spiculated margins inside the left breast b-b'-b") Axial T1 MRI image: pre-cryoablation image shows enhancing lesion at the same site
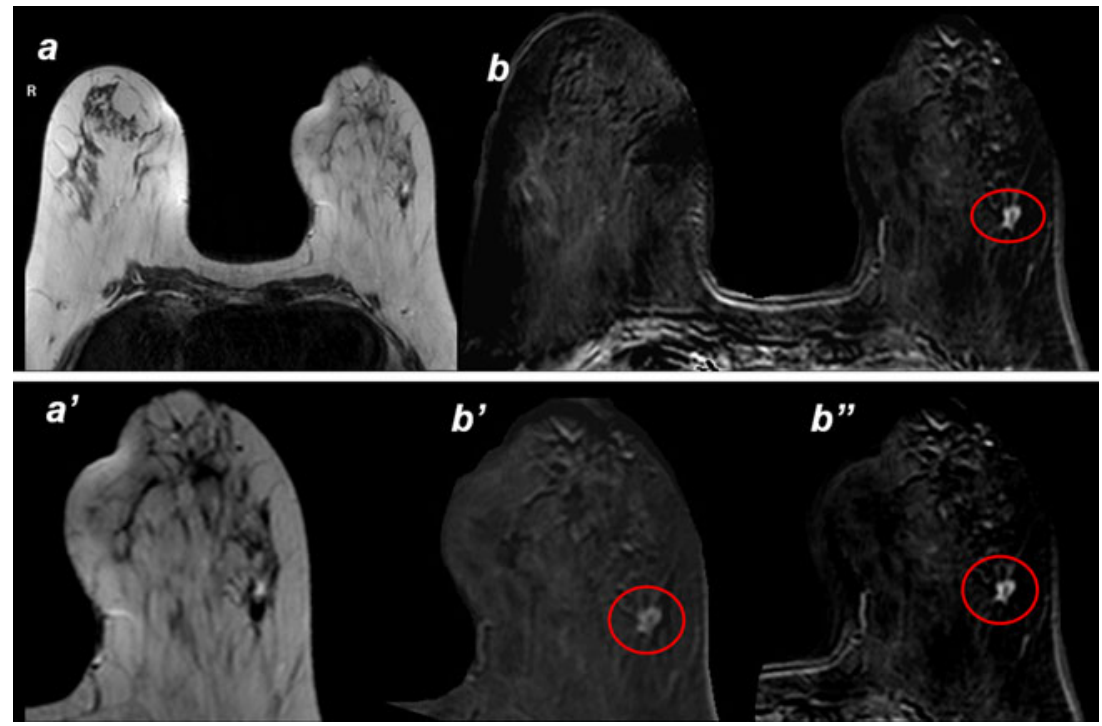
Fig. 5 Axial T1 (a) and T2 (a') pre-contrast MRI image; axial

T1 (b) post-contrast MRI image: one month after cryoablation the necrosis area is clearly visible (a, a') and the enhancing lesion has disappeared (b)
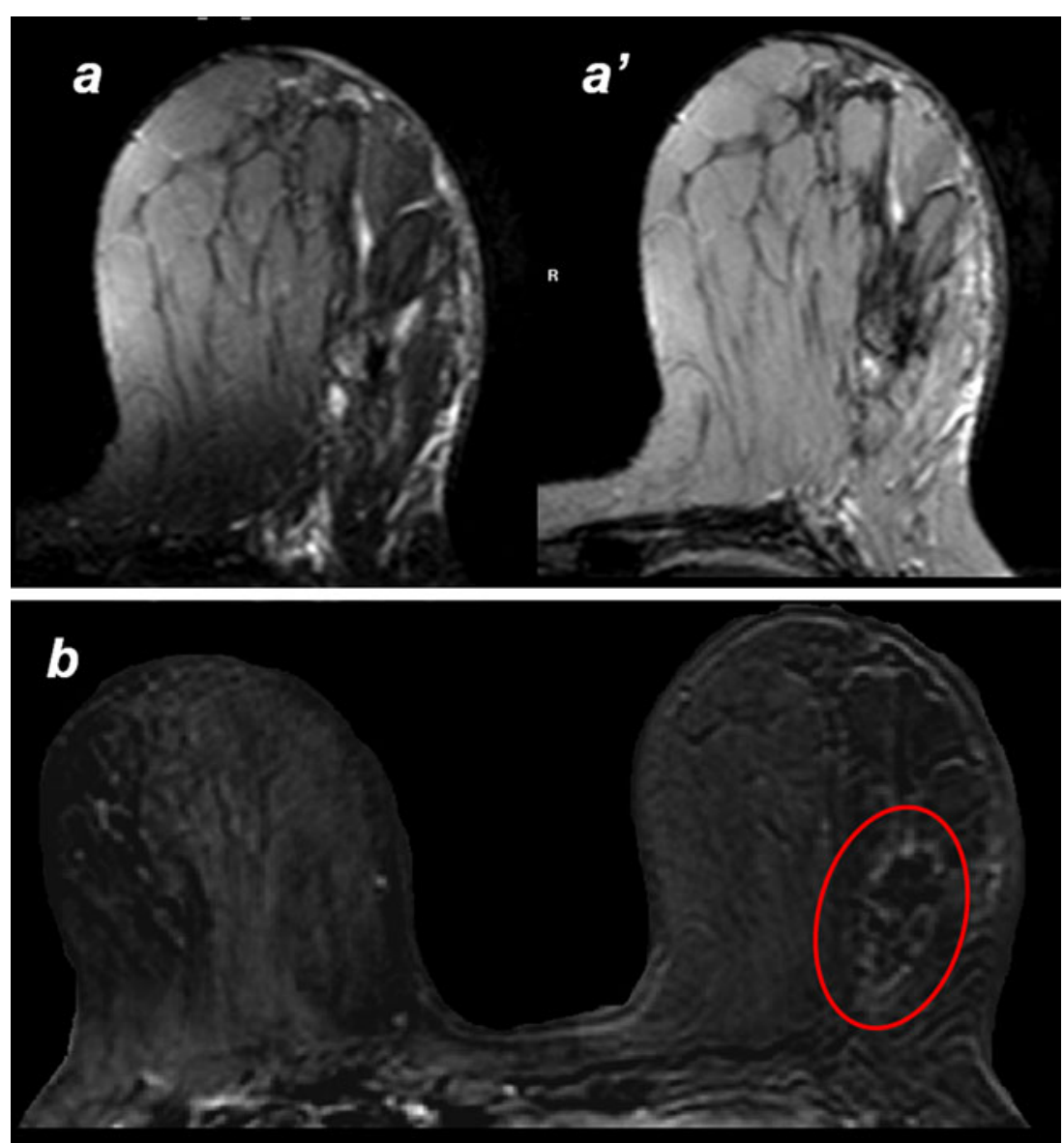

Wilcoxon test for data non-parameters. The analysis of correlation were calculated Coefficient of Pearson (r). Statistical significance was set at a $\mathrm{p}$ value $<0.05$.

\section{Results}

The pre-ablation US-guided vacuum assisted biopsy (VAB) of the breast in 15 treated patients revealed all invasive ductal carcinoma tumours. Of these, 13 were endocrine responsive (ER, PR, HER2-), 1 was not endocrine responsive (ER-, PR-, HER2) and 1 was a triple negative (ER-, PR-,HER2-). Twelve out of 15 were well differentiated
(G1), 3 out of 15 were moderately differentiated (G2). All the neoplasia were endocrine responsive (ER and PR positive, Her-2 negative) with low proliferative index (Ki-67 percentage ranged from 5 to $10 \%$ ). All lesions, measuring between 4 $12 \mathrm{~mm}$, were treated by cryo-probes and lumpectomy. The iceball size around the probe at $-40^{\circ} \mathrm{C}$ was $16 \times 41 \mathrm{~mm}$, as assessed by ultrasound at the end of the second cooling cycle. In 14 of the fifteen patients, we observed the complete necrosis of the cryoablated lesion in both postprocedural MRI and histopathology, with necrosis diameters shown in Table 1. Lesions volume (cubic $\mathrm{cm}$ ) observed both on post-ablative pre-operative MRI (MRI necrosis, $C$ ) and after surgical removal of the
Fig. 6 MR images show visualization and segmentation of the cryoablation-induced lesion in three perpendicular planes (left to right: axial, sagittal, coronal)

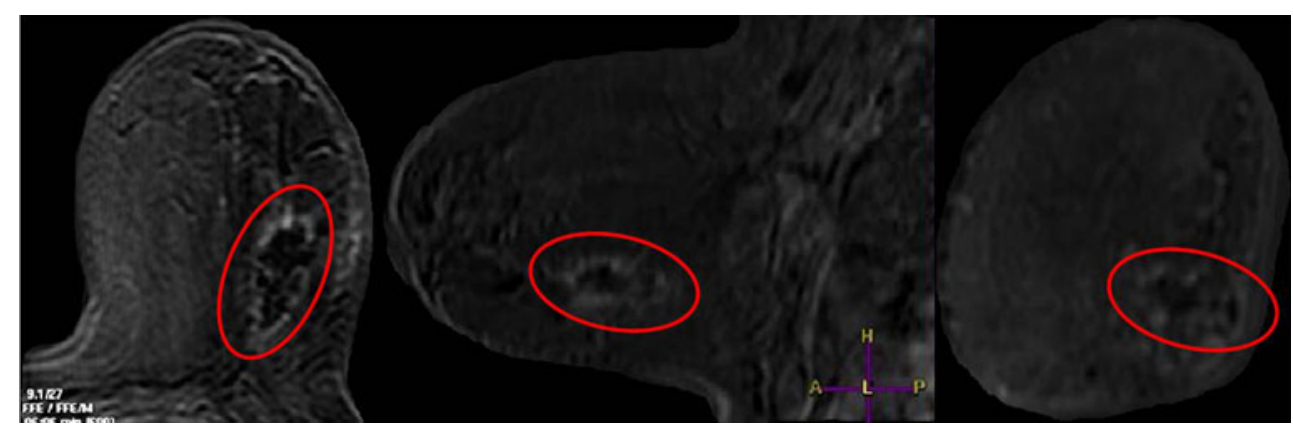


Table 1 The necrosis volume compilation was compared between the pre-surgical post-ablative gadolinium dynamic MRI images and the pathologist calipering on the fresh stained surgical specimen, considered as the gold standard. Data are expressed in terms of lesions volume $(\mathrm{cm} 3)$ observed both on post-ablative pre-operative MRI (MRI necrosis) and after surgical removal of the necrosis itself (histological necrosis)

\begin{tabular}{lll}
\hline Patients & $\begin{array}{l}\text { MRI necrosis }\left(\mathrm{cm}^{3}\right) \\
\text { C' }\end{array}$ & $\begin{array}{l}\text { Histological necrosis }\left(\mathrm{cm}^{3}\right) \\
\mathrm{C}^{\prime}\end{array}$ \\
\hline 1 & 25,32 & 13,76 \\
2 & 29,89 & 12,05 \\
3 & 11,55 & 4,29 \\
4 & 17,58 & 8,70 \\
5 & 25,32 & 16,17 \\
6 & 45,21 & 21,22 \\
7 & 7,03 & 2,49 \\
8 & 34,96 & 14,96 \\
9 & 42,70 & 24,38 \\
10 & 28,89 & 20,41 \\
11 & 24,49 & 13,06 \\
12 & 29,89 & 19,69 \\
13 & 28,13 & 28,82 \\
14 & 42,20 & 25,30 \\
15 & 35,23 & 33,76 \\
mean $\infty \pm$ SD & $28,56 \pm 10,90 *$ & $17,27 \pm 8,77$ \\
\hline
\end{tabular}

*MRI vs Histological necrosis $t$-test; $p<0.001$

Histological necrosis $=f(\mathrm{MRI})$; coorelation coefficient $r=0.769$

necrosis itself (histological necrosis, $C^{\prime}$ ) were statistically different ( $t$-test, $\mathrm{df}=14 ; p<0,001)$ (Table 1$)$. The coefficient of correlation between these two measures was $r=0.769$ (Pearson coefficient, $p<0.001$ ).

In one case at post-procedural MRI there was a decentralized ablation according to the core of the lesion, probably justified by an inappropriate positioning of the probe during the procedure because of patient poor compliance. MRI examinations with dynamic enhanced sequences, showed the absence of residual angiogenesis in 14 of the 15 patients as like as the absence of vital neoplastic cells at haematoxylin-eosin coloration. In the last patient, MRI showed contrast residual uptake with an enhanced outbreak of about $3 \mathrm{~mm}$, as an index of residual active disease. In the patient with partial necrosis at haematoxylin-eosin coloration, residual cell clusters of glandular tissue cancer adjacent to the ablation spot were noticed. Interpretation of lymph node mapping and subsequent identification and removal of the sentinel node was not altered in any case because of percutaneous ablation. Cryo-lumpectomy was well tolerated by patients with only i.v. sedation for about $30 \mathrm{~min}$ of procedure time. Postprocedural pain requiring narcotic pain-relieving drugs was never experienced. Onset of subcutaneous haematoma in two cases as long as post-procedural complication. The sentinel node histology was always negative for neoplastic seedings.

Pathologic validation procedure

The morphological analysis did not show any residual neoplasia. In 14 out of 15 samples, the histological examination showed the presence of extensive areas of fibrosis, associated with a chronic granulomatous inflammatory reaction including hemosiderin deposits (previous hemorrhage) at the site of the cryoablation procedure. In 2 out of 15 samples, a chronic granulomatous inflammated area at the location of the probe was evidenced. The immunohistochemistry reaction with anti-cytokeratins, carried out on all the cryoablated area sections, confirmed the absence of residual cancer in all cases except than in a single case were few residual cancer cells were associated with chronic eccentric inflammation along the tumour area probably due to eccentric positioning of the probe.

Post-procedural cosmetics assessment

The following signs at the end of the ablation procedure (Time 0 ) and at 1 month from the procedure, prior to the surgical resection of the tumour (Time I) were collected (Table 2): in 12 of the 15 patients, we detected the presence of a skin rash (Fig. 7) (which resolved spontaneously within $8 \mathrm{~h}$ ) in 5 cases associated with the presence of a clinically appreciable nodular thickening and in 4 cases the presence of skin bruising; in 1 patient we detected the presence of a nodular thickening at the procedure area; in 2 cases no sign was observed.

\section{Discussion}

Cryotherapy is based on the use of cryoablation needles, which extracting heat from the tissue determine cooling and subsequent freezing. The cryoablation protocol depends on the necrosis volume to induce obtaining subsequent freezing/heating cycles. The use of multiple needles and cycles can accelerate and expand the phenomenon, providing treatment for different size injuries [5]. Currently, by using a gas expansion system, can be constructed even thinner needles than the ones by liquid nitrogen applied so far (actual needle diameter of $1.47 \mathrm{~mm}$ against the needles with liquid nitrogen diameter of 3-3.5 mm), while maintaining the needle temperature in a range of around $-180^{\circ} \mathrm{C}$. Furthermore, the technique of gas expansion allows keeping both cooling and heating of the same needle by using two gasses (argon and helium) of different thermodynamic properties. This is reflected in a lot of practical advantages, 
Table 2 The post-ablation cosmetics was assessed respectively at the end of the ablation (Time 0 ) and at 1 month from the procedure, prior to the surgical resection of the tumor (Time I). The cosmetic assessment was expressed according to the presence or absence of 4 parameters: appreciable nodular thickening on palpation (a); skin bruising (b); skin rash (c); skin hyperpigmentation (d). Some of the signs found at T0 regressed spontaneously without therapy; others persisting the same at the TI observation, or modified as permanent cosmetic changes. At TI, in the 5 cases associated with skin rash and clinically appreciable nodular thickening, a permanent nodular thickening was observed in 2 cases, it was the only case of nodular thickening at the procedure's site constantly palpable up to the surgical excision; in 3 cases of the skin bruising appearance, the appearance of a skin hyperpigmentation area was recorded

\begin{tabular}{|c|c|c|}
\hline Patients & Time 0 (after-ablation) & Time I (pre-surgical) \\
\hline 1 & $\mathrm{~b}+\mathrm{a}$ & \\
\hline \multicolumn{3}{|l|}{2} \\
\hline 3 & $\mathrm{~b}$ & \\
\hline 4 & $\mathrm{~b}$ & \\
\hline 5 & $\mathrm{~b}+\mathrm{c}$ & $\mathrm{d}$ \\
\hline 6 & $\mathrm{~b}+\mathrm{a}$ & \\
\hline \multicolumn{3}{|l|}{7} \\
\hline 8 & $\mathrm{~b}+\mathrm{a}$ & $\mathrm{a}$ \\
\hline 9 & $\mathrm{a}$ & $\mathrm{a}$ \\
\hline 10 & $\mathrm{~b}+\mathrm{c}$ & $\mathrm{d}$ \\
\hline 11 & $\mathrm{~b}$ & \\
\hline 12 & $b+a$ & \\
\hline 13 & $\mathrm{~b}+\mathrm{a}$ & $\mathrm{a}$ \\
\hline 14 & $b+c$ & \\
\hline 15 & $b+c$ & $\mathrm{~d}$ \\
\hline
\end{tabular}

particularly allowing reduced treatment length. In our survey we used a cooling double-cycle (each $10 \mathrm{~min}$ ) separated by a 5 min of passive heating (stop of argon coolant flow) and an active heating with helium of the cryoprobe, after the second cooling cycle, in order to achieve complete cell necrosis in much lower than zero $\left(-40^{\circ} \mathrm{C}\right)$ temperatures. At this temperature, the exposed cells are destroyed by the intra- and extra- cellular ice formation, depending on the cooling rate. The cooling/heating double-cycle increases the probability of intracellular ice formation and subsequent direct cell damage. We operated in all the cases "high freeze" cycles in which argon supplies the system for all the duration of the cooling cycle $(100 \%$ duty cycle) with a progressive size increase of the iceball until the maximum possible size. The cells survived from the direct freezing, die of ischemia and structural damage (delayed cell damage). In the current practice, the radiofrequency ablation (RF) system is the most useful and promising technique among all the applied techniques of percutaneous ablation for the treatment of breast cancer smaller than $2 \mathrm{~cm}[6,7]$. Problems and limitations related to the use of radiofrequency as a physical principle of percutaneous breast cancer ablation are largely attributable to insufficient tumour ablation, to the lack of a real-time imaging that allows to effectively monitor the procedure, to possible side effects on the skin, to the literature absence of significant samples of patients and/or sensitive distant follow-up. "Incomplete" and/or "inconclusive" ablations were attributed to technical difficulties such as probe positioning bias, system malfunctions, and even to the underestimation of pre-procedural imaging of tumour extension. In our study, the use of high field MRI (3 Tesla) in the patients selection probably removed the bias related to the "underestimation of disease". Unsolved problem with breast cancer percutaneous radiofrequency ablation is the need to monitor the ablation in real-time. If the extensive intraprocedural gas produced by the $\mathrm{RF}$ coagulation necrosis does not permit the core ablation view, resulting in lesion re-centering difficulties, the cryoprobe positioning seems to be much more simplified with real-time ultrasound. Percutaneous cryoablation of subclinical breast cancer can be considered an effective ablation technique, repeatable, painless and at low morbidity. In particular, it became clear the analgesic effect of coldness, compared to radiofrequency [7]. In terms of cosmetics, cryoablation represents many significant
Fig. 7 The post-procedural cosmetics evaluation (Time 0 ) shows the presence of a skin rash on the site of the cryoablation procedure
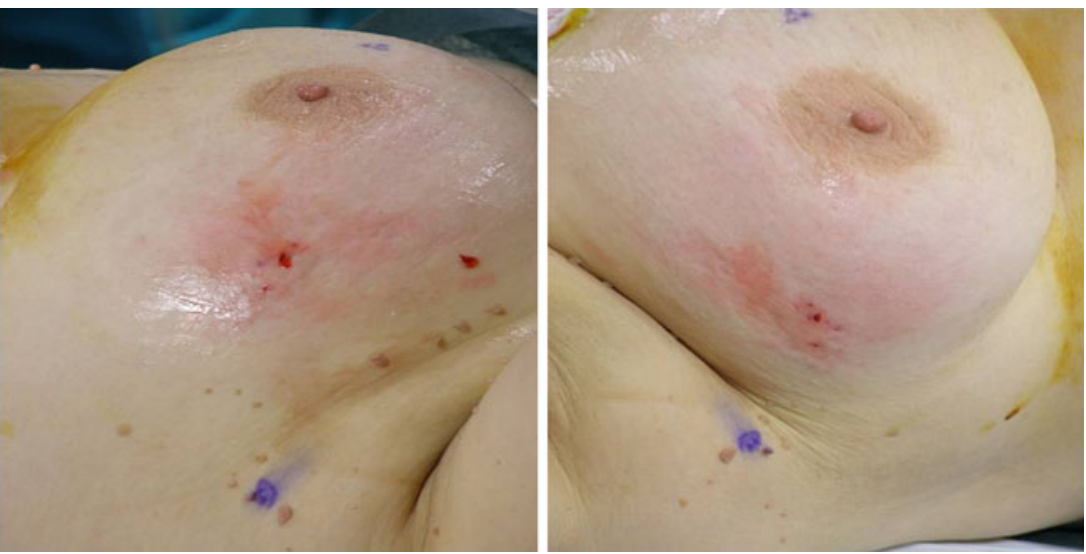
advantages compared to RF thermoablation [8] We obtained optimal results in 9 patients, in whom no sign was observed, and good results in 6 patients, with signs of slight hyperpigmentation persistent in 3 patients and mild nodular thickening in 3 other patients. The fully recover was also moderately faster and significantly more complete. A bias related to the radiofrequency use is probably associated with a common degree of perilesional liponecrosis with consequent phenomenons of skin retraction in small dimension breasts and/or voluminous mammary lesions. Regarding the complications, the post-procedural discomfort, has remained limited in all cases, due to breast tension sensation, swelling, transient bruising and sometimes "palpable mass". In 5 cases, with the tumour $1 \mathrm{~cm}$ far away from the skin profile, sterile saline $(20-30 \mathrm{~mL})$ heated solution to $40^{\circ}$ was injected between the lesion and the skin, to avoid damaging the surrounding skin. In our experience and literature data emerge some absolute contraindications of cryoablation use in percutaneous breast cancer treatment. As it is indicated in the treatment of isolated and confined lesions, entirely viewable by ultrasound, patients with extensive intraductal component and/or invasive lobular carcinoma and/or multifocal or multicentric tumours are not candidates to percutaneous ablative treatment. Although it has been reported in literature the ablation possibility of breast tumours up to $4.5 \mathrm{~cm}$ in diameter, patients with focal lengths greater than $2 \mathrm{~cm}$ are not candidates to this treatment, due to the presence of "hidden" intra-ductal branching cancer and DCIS (ductal carcinoma in situ) beyond the perifocal safety margin guaranteed by this technique. The simultaneous use of multiple probes, resulting in exponential summation of necrosis volume represents a questionable procedural complication in this organ application [9] . In literature there are numerous studies on invasive breast cancer cryoablation characterized by different selection criteria and results [9-12]. The extent of microcalcification visible on mammography often underestimates the true extention of carcinoma in situ and this is further restricted by the selection of patients to be treated. The persistence of carcinoma in situ adjacent to the ablated lesion [13], still incapsuled into subtle perilesional ramifications, is seen more frequently on lesions with dimensions $>1.5 \mathrm{~cm}$ and DCIS $>25 \%$. DCIS is more frequently located peripherally to the neoplastic lesion, where developed temperatures are less effective because non-lethal. Based on these considerations, percutaneous breast cancer ablation techniques should be carefully avoided in DCIS, representing an ideal technique for tumours below 15$16 \mathrm{~mm}$ in diameter. Experiences with excellent results for breast expansion of significantly greater dimensions are reported for the fibroadenomas [14, 15] .The main disadvantage of in situ breast cancer ablation techniques not followed by surgical resection consists on the impossibility to assess anatomo-pathological changes, the excisional surgical specimen, the extent and completeness of ablation. Literature reported a cryotherapy experience not followed by surgical excision performed on animals [16]. In the opinion of the authors, although the percutaneous tumour ablation could lead to a partial tumour response, the adjuvant radiotherapy is used to clean up residual cancer cells. Currently, no diagnostic tool ensures optimal demonstration and follow-up of the in situ ablation, so we can consider this method as primary therapy for breast cancer. Recently, a study [17] showed the feasibility of multiprobe percutaneous breast cryoablation (BC) for different presentations of cancers, that remained in situ after BC, without surgery excision and with MR imaging and/or clinical follow-up available for up to 72 months after BC. In our study, correlating the ablated volume or the obtained necrosis, through the dimensional data calculated by MRI and the one calculated by histological sample, we noted a dimensional overestimation on MRI assessment in comparison with histopathology. In the MRI post-procedural evaluation, in one case, we observed residual angiogenesis or an enhancement outbreak of about $3 \mathrm{~mm}$, remaining from the disease. The problem of correct positioning of ablation catheter within the target lesion, which is currently entrusted exclusively to the operator's ability, in the future can be easily overcome by the use of new methods of real-time ultrasound image fusion and static magnetic resonance called "medical GPS" (electromagnetic tracking). Therefore, the 3D injury assessment associated with ultrasound data and operator's experience, may help to achieve a greater volume of necrosis though it can correct the probe positioning defects.

In conclusion we can reasonably assume that there are real in vivo possibilities, on selected and handled cases by experienced operators, to achieve optimal cosmetic and oncological results using in situ minimally invasive ablation techniques with tested devices.

\section{References}

1. Veronesi U, Cascinelli N, Mariani L (2002) Twenty-years followup of a randomized study comparing breast-conserving surgery with radical mastectomy for early breast cancer. N Engl J Med 347:1227-32

2. Fisher B, Anderson S, Bryant J (2002) Twenty-years follow-up of a randomized study comparing total mastectomy, lumpectomy, and lumpectomy plus irradiation for the treatment of invasive breast cancer. N Engl J Med 347:1227-32

3. Kimmick GG, Balducci L (2000) Breast cancer and aging. Clinical interactions. Hematol Oncol Clin North Am 14:213-34

4. Parkin DM, Bray FI, Devesa SS (2000) Cancer burden in the year 2000. The global picture. Eur J Cancer 37:S4-S66 
5. Littrup PJ, Jallad B, Vorugu V (2009) Lethal isotherms of cryoablation in a phantom study: effects of heat load, probe size and number. J Vasc Interv Radiol 20:1343-1351

6. Fornage BD, Sneige N (2004) Small $(\leq 2 \mathrm{~cm})$ breast cancer treated with US-guided radiofrequency ablation: feasibility study. Radiology 231:215-224

7. Manenti G, Bolacchi F, Perretta T (2009) Small breast cancers: in vivo percutaneous US-guided radiofrequency ablation with dedicated cool-tip radiofrequency system. Radiology 251:339-46

8. Vlastos G, Verkooijen H (2007) Minimally invasive approaches for diagnosis and treatment of early-stage breast cancer. Oncologist 12:1-10

9. Pfleiderer SO, Freesmeyer MG, Marx C (2002) Cryotherapy of breast cancer under ultrasound guidance: initial results and limitations. Eur Radiol 12:3009-14

10. Pfleiderer SO, Marx C, Camara O (2005) Ultrasound-guided percutaneous cryotherapy of small $(\leq 15 \mathrm{~mm})$ breast cancers. Invest Radiol 40:472-477
11. Sabel MS, Kaufman CS, Whitworth P (2004) Cryoablation of early-stage breast cancer: work-in-progress report of a multiinstitutional trial. Ann Surg Oncol 11:542-49

12. Morin J, Traore A, Dionne G (2004) Magnetic resonance-guided percutaneous cryosurgery of breast carcinoma: technique and early clinical results. Can J Surg 47:347-51

13. Pusztaszeri M, Vlasos G, Kinkel K (2007) Histopathological study of breast cancer and normal breast tissue after magnetic resonance-guided cryotherapy ablation. Criobiology 55:44-51

14. Kaufman CS, Littrup PJ, Freeman-Gibb LA (2005) Office-based cryoablation of the breast fibroadenomas with long-term followup. Breast J 11:344-50

15. Nurko J, Mabry CD, Whitworth P (2005) Interim results from the fibroadenoma cryoablation registry. Am J Surg 190:647-51

16. Staren ED, Sabel MS, Gianakakis LM (1997) Cryosurgery of breast cancer. Arch Surg 132:28-33

17. Littrup PJ, Jallad B, Chandiwala-Mody P (2009) Cryotherapy for breast cancer: a feasibility study without excision. J Vasc Interv Radiol 20:1329-13410 